\title{
Eliminating Decoherence Effects and Improving Reconstructed Image Quality in Soft X-ray Ptychography.
}

\author{
Chunpeng Wang, Zijian Xu$*$, Haigang Liu, Xulei Tao and Renzhong Tai \\ Shanghai Synchrotron Radiation Facility, Shanghai Institute of Applied Physics, CAS, Shanghai, China \\ * Corresponding author, xuzijian@sinap.ac.cn
}

Ptychography is a diffraction-based X-ray microscopy method that can image extended samples quantitatively and can remove the resolution limit imposed by image-forming optical elements [1,2]. In most ptychography reconstruction algorithms, the incident X-ray is considered to be fully coherent and monochromatic (i.e. single pure state), the sample is considered to be stable or unchanged, and the responses of all optical elements in the system are considered to be fixed throughout the data acquisition process. However, in actual X-ray ptychography experiments, it is almost impossible to satisfy all the above conditions at the same time. Under non-ideal conditions, decoherence effects could severely decrease the fringe visibility in the diffracted patterns and deteriorate the reconstructed sample and probe images [3]. It is a challenging issue to eliminate the negative decoherent effects from various sources in practical ptychography experiments and thus improve reconstructed image quality. Recent developments in ptychography have extends to mixed states approaches, for example multiple probe states retrieved when the illumination is partially coherent or multiple object states when laterally vibration occurs, relying on orthogonal decomposition of the probe and/or the object coherent states $[3,4]$. These methods not only substantially relax the requirements on experiments, but also further extend the applications of ptychography. During our processing of experimental data, we found another algorithm, the sub-pixel up-sampling method [5], shows a comparable ability to the mixed states method in eliminating the partial coherence effects. Therefore, we compare the two methods in this paper.

The soft X-ray ptychography experiment was carried out at BL08U1A beamline of Shanghai Synchrotron Radiation Facility [6-8]. A partially coherent X-ray beam was produced from the monochromator exit slit which is at $2 \mathrm{~m}$ upstream of the Fresnel zone plate (FZP). The size of the exit slit was $50 \times 50 \mu \mathrm{m}$ which produced transverse coherent lengths of about $30(\mathrm{H}) \times 50(\mathrm{~V}) \mu \mathrm{m}$ at $700 \mathrm{eV}$. The $200 \mu \mathrm{m}$ diameter FZP with a $80 \mu \mathrm{m}$ diameter central stop and $30 \mathrm{~nm}$ outermost zone width in combination with a $70 \mu \mathrm{m}$ diameter order sorting aperture was placed downstream of the X-ray exit window. A Siemens star test pattern with the finest stripe width of $30 \mathrm{~nm}$ was placed $51.4 \mu \mathrm{m}$ downstream of the FZP focus. With $708 \mathrm{eV}$ incident X-ray, a probe size of about $3 \mu \mathrm{m}$ was obtained. Far field diffraction patterns were recorded using a CCD detector which was at $74 \mathrm{~mm}$ downstream of the sample. Using a $1200 \times 1200$ array of the CCD with a $13.5 \mu \mathrm{m}$ pixel size, the reconstructed object pixel size was $8 \mathrm{~nm}$. A $10 \times 10$ raster grid was scanned across the test pattern with a $500 \mathrm{~nm}$ step size.

The reconstructed amplitude images of the test pattern using three different algorithms: single state ePIE [9], mixed states ePIE with five probe states, and sub-pixel up-sampling ePIE (sPIE) with a $3 \times 3$ upsampling ratio, are shown in Figure 1(a-c), respectively, and compared with an SEM image Figure 1(d). Note that evident contamination exists in the central area of the star pattern, resulting from long-term exposure to atmosphere and long-time irradiation by X-ray. Considering both the probe and the sample as a single state, Figure 1(a) shows the worst image quality. The $30 \mathrm{~nm}$ stripes in the center region are blurred and almost unseen, and the intensity distribution inside and outside the $60 \mathrm{~nm}$ stripes in the peripheral region is uneven. In contrast, the reconstruction quality is greatly improved in Figure 1(b), 
indicating the negative effects of partial coherence are substantially eliminated, which is predictable because the illuminating probe is partially coherent due to the exit slit settings. What is surprising is that the sPIE result Figure 1(c) also shows a great quality improvement, which is comparable to that of the mixed states ePIE.

The reconstructed amplitude images of probe functions by the three algorithms are shown in Figure 2 . As shown in Figure 2(a), the probe function reconstructed by single state ePIE is severely deviated from what it should be under ideal conditions (a pure single state), which in turn echoes the poor quality of the reconstructed sample image. The five reconstructed probe functions using mixed states ePIE in Figure 2(b-f) are much cleaner than that in Figure 2(a), but there are high-intensity irregular spots in the corners of each probe function image, which is probably from some kind of strong experimental noise other than partially coherent probe. The reconstructed probe function (Figure 2(g)) by sPIE shows several spatially isolated probe-like distributions in real space (sample plane) outside the real probe area. It seems that these probe-like distributions in sPIE play a similar role in the frequency domain as the multiple-mode probes in mixed states ePIE. Therefore, we think that the sPIE algorithm probably has an equivalent capability of eliminating partial coherence effects as the mixed states method, which enables sPIE to provide high quality reconstructed images under partially coherent illuminations.

\section{References:}

[1] H. M. L. Faulkner and J. M. Rodenburg, Physical Review Letters 93 (2004) 023903.

[2] P. Thibault et al, Science 321 (2008) 379-382.

[3] P. Thibault and A. Menzel, Nature 494 (2013) 68-71.

[4] P. Li et al, Optics Express 24(6) (2016) 9038-9052.

[5] D. J. Batey et al, Physical Review A 89 (2014) 043812.

[6] C. Wang et al, Nuclear Science and Techniques 28 (2017) 74.

[7] C. Wang et al, Applied Optics 56(8) (2017) 2099-2111.

[8] Z. Xu et al, Journal of Physics: Conference Series, 849(1) (2017) 012033.

[9] A. M. Maiden and J. M. Rodenburg, Ultramicroscopy 109 (2009) 1256-1262.

[10] The authors acknowledge funding from the National Natural Science Foundation of China (grant Nos. 11705273, 11575283, 11505277)

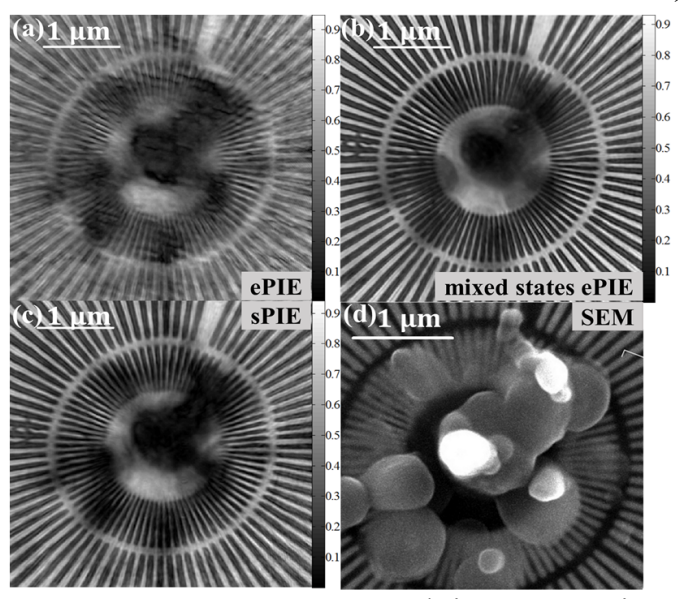

Figure. 1. Reconstructed images using three different algorithms: ePIE (a), mixed states ePIE (b), and SPIE (c), compared with an SEM image (d).

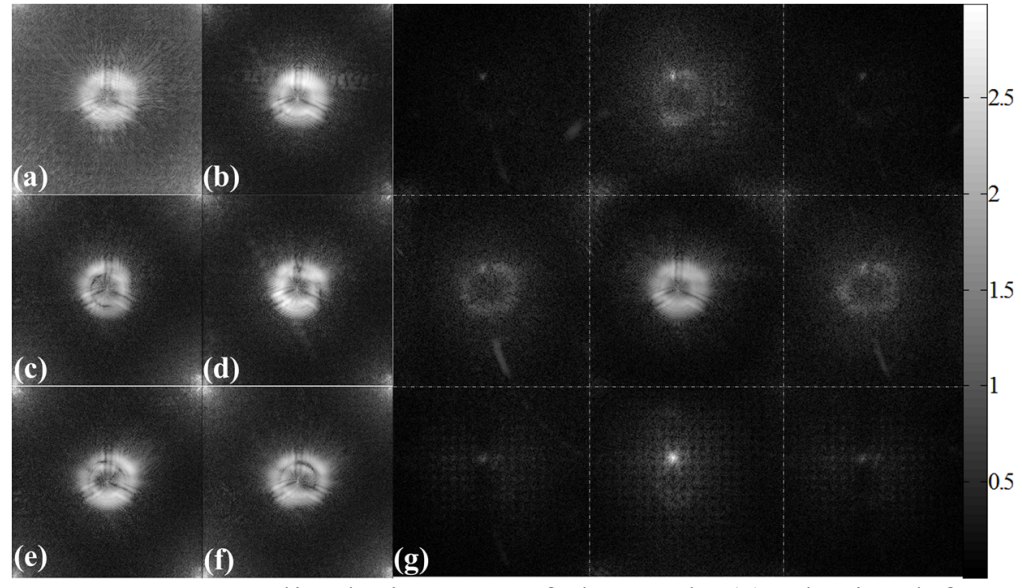

Figure. 2. Amplitude images of the probe(s) obtained from different algorithms: (a) ePIE, (b-f) mixed states ePIE, (g) sPIE. All images are displayed in logarithmic scale. 